DEMONSTRATIO MATHEMATICA

Vol. XLII No 42009

Servet Kutukcu, Sushil Sharma

\title{
A COMMON FIXED POINT THEOREM IN NON-ARCHIMEDEAN MENGER PM-SPACES
}

\begin{abstract}
In the present work, we introduce two types of compatible maps in nonArchimedean Menger PM-spaces and obtain a common fixed point theorem for six maps.
\end{abstract}

\section{Introduction and preliminaries}

In 1997, Cho et al. [2] introduced the concepts of compatible maps and compatible maps of type (A) in non-Archimedean Menger probabilistic metric spaces and gave some fixed point theorems for these maps. In this paper, we introduce the concept of compatible maps of type (A-1) and type (A-2), show that they are equivalent to compatible maps under certain conditions and illustrating with an example, prove a common fixed point theorem for such maps in the spaces which generalizes, extends and fuzzifies several fixed point theorems for contractive type maps on metric spaces and fuzzy metric spaces.

Next, we recall some definitions and known results in Menger space. For more details we refer the readers to [1-7].

Definition 1. A triangular norm * (shorty t-norm) is a binary operation on the unit interval $[0,1]$ which is associative, commutative, nondecreasing in each coordinate and $a * 1=a$ for all $a \in[0,1]$. Some important examples of t-norms are $a * b=\max \{a+b-1,0\}$ and $a * b=\min \{a, b\}$.

Definition 2. A distribution function is a function $F:[-\infty, \infty] \rightarrow[0,1]$ which is left continuous on $\mathbb{R}$, non-decreasing and $F(-\infty)=0, F(\infty)=1$. If $X$ is a nonempty set, $F: X \times X \rightarrow \Delta$ is called a probabilistic distance on $X$ and $F(x, y)$ is usually detoned by $F_{x y}$.

2000 Mathematics Subject Classification: 47H10, 54H25, 54E70.

Key words and phrases: non-Archimedean Menger probabilistic metric space, compatible maps, mutually compatible maps, common fixed point. 
Definition 3. The ordered pair $(X, F)$ is called a non-Archimedean probabilistic metric space (shortly N. A. PM-space) if $X$ is a nonempty set and $F$ is a probabilistic distance satisfying the following conditions: for all $x, y, z \in X$ and $t, s \geq 0$,

(PM-1) $F_{x y}(t)=1, t>0 \Longleftrightarrow x=y$,

(PM-2) $F_{x y}=F_{y x}$

(PM-3) $F_{x y}(0)=0$,

$(\mathrm{PM}-4) F_{x y}(t)=1, F_{y z}(s)=1 \Rightarrow F_{x z}(\max \{t, s\})=1$.

The ordered triple $(X, F, *)$ is called a non-Archimedean Menger probabilistic metric space (shortly N. A. Menger space) if $(X, F)$ is a N. A. PM-space, $*$ is a t-norm and the following condition is also satisfies: for all $x, y, z \in X$ and $t, s>0$,

$(\mathrm{PM}-5) F_{x z}(\max \{t, s\}) \geq F_{x y}(t) * F_{y z}(s)$.

The concept of neighbourhoods in Menger PM-spaces was introduced by Schweizer and Sklar [6]. If $x \in X, \varepsilon>0$ and $\lambda \in(0,1)$, then an $(\varepsilon, \lambda)$ neighbourhood of $x, U_{x}(\varepsilon, \lambda)$ is defined by

$$
U_{x}(\varepsilon, \lambda)=\left\{y \in X: F_{x y}(\varepsilon)>1-\lambda\right\} .
$$

If the t-norm $*$ is continuous and strictly increasing then $(X, F, *)$ is a Hausdorff space in the topology induced by the family $\left\{U_{x}(\varepsilon, \lambda): x \in X, \varepsilon>\right.$ $0, \lambda \in(0,1)\}$ of neighbourhoods $[6]$.

Definition 4. ([2]) A PM-space $(X, F)$ is said to be of type $(\mathrm{C})_{g}$ if there exists a $g \in \Omega$ such that

$$
g\left(F_{x y}(t)\right) \leq g\left(F_{x z}(t)\right)+g\left(F_{z y}(t)\right)
$$

for all $x, y, z \in X$ and $t \geq 0$ where $\Omega=\{g: g:[0,1] \rightarrow[0, \infty)$ is continuous, strictly decreasing, $g(1)=0$ and $g(0)<\infty\}$.

Definition 5. ([2]) A N. A. Menger PM-space $(X, F, *)$ is said to be of type (D) $g$ if there exists a $g \in \Omega$ such that

$$
g(t * s) \leq g(t)+g(s)
$$

for all $s, t \in[0,1]$.

Remark 1. If a N. A. Menger PM-space $(X, F, *)$ is said to be of type $(\mathrm{D})_{g}$ then $(X, F, *)$ is of type $(\mathrm{C})_{g}$. On the other hand, $(X, F, *)$ is a $\mathrm{N}$. A. PM-space such that $a * b \geq \max \{a+b-1,0\}$ for all $a, b \in[0,1]$, then $(X, F, *)$ is of type (D) $)_{g}$ for $g \in \Omega$ defined by $g(t)=1-t, t \geq 0$.

Throughout this paper, let $(X, F, *)$ be a complete N. A. Menger PMspace of type $(D)_{g}$ with a continuous strictly increasing t-norm * and 
$\phi:[0, \infty) \rightarrow[0, \infty)$ be a function satisfying the condition $(\Phi): \phi$ is uppersemicontinuous from the right and $\phi(t)<t$ for all $t>0$.

LEMMA 1. ([1]) If a function $\phi:[0, \infty) \rightarrow[0, \infty)$ satisfies the condition $(\Phi)$, then we have

(a) for all $t \geq 0, \lim _{n \rightarrow \infty} \phi^{n}(t)=0$ where $\phi^{n}(t)$ is the $n$-th iteration of $\phi(t)$,

(b) if $\left\{t_{n}\right\}$ is a non-decreasing sequence of real numbers and $t_{n+1} \leq \phi\left(t_{n}\right)$, $n=1,2, \ldots$ then $\lim _{n \rightarrow \infty} t_{n}=0$. In particular, if $t \leq \phi(t)$ for all $t \geq 0$ then $t=0$.

LEMMA 2. ([2]) Let $\left\{y_{n}\right\}$ be a sequence in $X$ such that $\lim _{n \rightarrow \infty} F_{y_{n}, y_{n+1}}(t)=$ 1 for all $t>0$. If $\left\{y_{n}\right\}$ is not a Cauchy sequence in $X$, then there exist $\varepsilon_{0}>0, t_{0}>0$ and two sequences $\left\{m_{i}\right\},\left\{n_{i}\right\}$ of positive integers such that

(a) $m_{i}>n_{i}+1$ and $n_{i} \rightarrow \infty$ as $i \rightarrow \infty$,

(b) $F_{y_{m_{i}}, y_{n_{i}}}\left(t_{0}\right)<1-\varepsilon_{0}$ and $F_{y_{m_{i}-1}, y_{n_{i}}}\left(t_{0}\right) \geq 1-\varepsilon_{0}, i=1,2, \ldots$

Definition 6. ([2]) Self maps $A$ and $B$ of a N. A. Menger PM-space $(X, F, *)$ are said to be compatible if $g\left(F_{A B x_{n} B A x_{n}}(t)\right) \rightarrow 0$ for all $t>0$, whenever $\left\{x_{n}\right\}$ is a sequence in $X$ such that $A x_{n}, B x_{n} \rightarrow z$ for some $z$ in $X$ as $n \rightarrow \infty$.

Definition 7. ([2]) Self maps $A$ and $B$ of a N. A. Menger PM-space $(X, F, *)$ are said to be compatible of type (A) if $g\left(F_{A B x_{n} B B x_{n}}(t)\right) \rightarrow 0$ and $g\left(F_{B A x_{n} A A x_{n}}(t)\right) \rightarrow 0$ for all $t>0$, whenever $\left\{x_{n}\right\}$ is a sequence in $X$ such that $A x_{n}, B x_{n} \rightarrow z$ for some $z$ in $X$ as $n \rightarrow \infty$.

Now we introduce the concept of compatible mappings of type (A-1) and type (A-2) in N. A. Menger PM-spaces and show that they are equivalent to compatible mappings under certain conditions.

Definition 8. Self maps $A$ and $B$ of a N. A. Menger PM-space $(X, F, *)$ are said to be compatible of type (A-1) if $g\left(F_{A B x_{n} B B x_{n}}(t)\right) \rightarrow 0$ for all $t>0$, whenever $\left\{x_{n}\right\}$ is a sequence in $X$ such that $A x_{n}, B x_{n} \rightarrow z$ for some $z$ in $X$ as $n \rightarrow \infty$.

Definition 9. Self maps $A$ and $B$ of a N. A. Menger PM-space $(X, F, *)$ are said to be compatible of type (A-2) if $g\left(F_{B A x_{n} A A x_{n}}(t)\right) \rightarrow 0$ for all $t>0$, whenever $\left\{x_{n}\right\}$ is a sequence in $X$ such that $A x_{n}, B x_{n} \rightarrow z$ for some $z$ in $X$ as $n \rightarrow \infty$.

REMARK 2. Clearly, if a pair of mappings $(A, B)$ is compatible of type (A-1) then the pair $(B, A)$ is compatible of type (A-2). Such maps are called mutually compatible of type (A). Further, if $A$ and $B$ compatible maps of type (A) then the pair $(A, B)$ is compatible of type (A-1) as well as type $(\mathrm{A}-2)$. 
The following is an example of pair of self maps in a N. A. Menger PM-space which are mutually compatible of type (A) but not compatible.

EXAMPLE 1. Let $(X, d)$ be a metric space with the usual metric $d$ where $X=[0,2]$ and $(X, F, *)$ be the induced N. A. Menger PM-space with $g(t)=$ $1-t$ and $F_{x y}(t)=H(t-d(x, y))$ for all $x, y \in X, t>0$. Define self maps $A$ and $B$ as follows:

$$
A x=\left\{\begin{array}{ll}
2-x, & \text { if } 0 \leq x<1, \\
2, & \text { if } 1 \leq x \leq 2,
\end{array} \text { and } B x= \begin{cases}x, & \text { if } 0 \leq x<1 \\
2, & \text { if } 1 \leq x \leq 2\end{cases}\right.
$$

Take $x_{n}=1-1 / n$. Then $F_{A x_{n} 1}(t)=H(t-(1 / n))$ and $\lim _{n \rightarrow \infty} g\left(F_{A x_{n} 1}(t)\right)=$ $g(H(t))=0$. Hence $A x_{n} \rightarrow 1$ as $n \rightarrow \infty$. Similarly, $B x_{n} \rightarrow 1$ as $n \rightarrow \infty$. Also $F_{A B x_{n} B A x_{n}}(t)=H(t-(1-1 / n))$ and $\lim _{n \rightarrow \infty} g\left(F_{A B x_{n} B A x_{n}}(t)\right)=$ $g(H(t-1)) \neq 0$ for all $t>0$. Hence the pair $(A, B)$ is not compatible. But $F_{A B x_{n} B B x_{n}}(t)=H(t-(2 / n))$ and $\lim _{n \rightarrow \infty} g\left(F_{A B x_{n} B B x_{n}}(t)\right)=g(H(t))=0$ for all $t>0$. Hence the pair $(A, B)$ is compatible of type (A-1). Similarly, the pair $(A, B)$ is compatible of type (A-2). Therefore $A$ and $B$ are mutually compatible but not compatible maps.

Next, we cite the following propositions which gives the condition under which the Definitions 6,8 and 9 becomes equivalent.

Proposition 1. Let $A$ and $B$ be self maps of a N. A. Menger PM-space $(X, F, *)$.

(a) If $B$ is continuous then the pair $(A, B)$ is compatible of type (A-1) iff $A$ and $B$ are compatible.

(b) If $A$ is continuous then the pair $(A, B)$ is compatible of type (A-2) iff $A$ and $B$ are compatible.

Proof. (a) Let $\left\{x_{n}\right\}$ be a sequence in $X$ such that $A x_{n}, B x_{n} \rightarrow z$ for some $z$ in $X$ as $n \rightarrow \infty$ and let the pair $(A, B)$ be compatible of type (A-1). Since $B$ is continuous, we have $B A x_{n} \rightarrow B z$ and $B B x_{n} \rightarrow B z$ and so

$$
g\left(F_{A B x_{n} B A x_{n}}(t)\right) \leq g\left(F_{A B x_{n} B B x_{n}}(t)\right)+g\left(F_{B B x_{n} B A x_{n}}(t)\right) \rightarrow 0
$$

as $n \rightarrow \infty$. Hence the mappings $A$ and $B$ are compatible.

Now, let $A$ and $B$ be compatible. Therefore, using the continuity of $B$, we have

$$
g\left(F_{A B x_{n} B B x_{n}}(t)\right) \leq g\left(F_{A B x_{n} B A x_{n}}(t)\right)+g\left(F_{B A x_{n} B B x_{n}}(t)\right) \rightarrow 0
$$

as $n \rightarrow \infty$. Hence the mappings $A$ and $B$ are compatible of type (A-1).

(b) The proof is similar with (a).

Next, we give some properties of compatible mappings of type (A-1) and type (A-2) which will be used in our main theorem. 
Proposition 2. Let $A$ and $B$ be self maps of a N. A. Menger PM-space $(X, F, *)$. If the pair $(A, B)$ is compatible of type (A-1) and $A z=B z$ for some $z$ in $X$ then $A B z=B B z$.

Proof. Let $\left\{x_{n}\right\}$ be a sequence in $X$ defined by $x_{n}=x$ for $n \in \mathbb{N}$ and let $A z=B z$. Then we have $A x_{n} \rightarrow A z$ and $B x_{n} \rightarrow B z$. Since the pair $(A, B)$ is compatible of type (A-1), we have $g\left(F_{A B z B B z}(t)\right)=g\left(F_{A B x_{n} B B x_{n}}(t)\right) \rightarrow 0$ as $n \rightarrow \infty$. Hence $A B z=B B z$.

Proposition 3. Let $A$ and $B$ be self maps of a N. A. Menger PM-space $(X, F, *)$. If the pair $(A, B)$ is compatible of type $(\mathrm{A}-2)$ and $A z=B z$ for some $z$ in $X$ then $B A z=A A z$.

Proof. The proof is similar with the proof of Proposition 2.

Proposition 4. Let $A$ and $B$ be self maps of a N. A. Menger PM-space $(X, F, *)$. If the pair $(A, B)$ is compatible of type (A-1) and $\left\{x_{n}\right\}$ is a sequence in $X$ such that $A x_{n}, B x_{n} \rightarrow z$ for some $z$ in $X$ as $n \rightarrow \infty$ then $B B x_{n} \rightarrow A z$ if $A$ is continuous at $z$.

Proof. Since $A$ is continuous at $z$ and the pair $(A, B)$ is compatible of type $(\mathrm{A}-1)$, we have $A B x_{n} \rightarrow A z$ and $g\left(F_{A B x_{n} B B x_{n}}(t)\right) \rightarrow 0$ as $n \rightarrow \infty$. Therefore

$$
g\left(F_{A z B B x_{n}}(t)\right) \leq g\left(F_{A z A B x_{n}}(t)\right)+g\left(F_{A B x_{n} B B x_{n}}(t)\right) \rightarrow 0
$$

as $n \rightarrow \infty$. Hence $B B x_{n} \rightarrow A z$ as $n \rightarrow \infty$.

Proposition 5. Let $A$ and $B$ be self maps of a N. A. Menger PM-space $(X, F, *)$. If the pair $(A, B)$ is compatible of type (A-2) and $\left\{x_{n}\right\}$ is a sequence in $X$ such that $A x_{n}, B x_{n} \rightarrow z$ for some $z$ in $X$ as $n \rightarrow \infty$ then $A A x_{n} \rightarrow B z$ if $B$ is continuous at $z$.

Proof. The proof is similar with the proof of Proposition 4.

\section{Main results}

Theorem 1. Let $A, B, P, Q, S$ and $T$ be self maps on a complete $N$. A. Menger PM-space $(X, F, *)$ satisfying:

(a) $P(X) \subseteq S T(X), Q(X) \subseteq A B(X)$,

(b) $g\left(F_{P x, Q y}(t)\right) \leq \phi\left(g\left(F_{A B x, S T y}(t)\right)\right)$,

(c)

$$
\begin{aligned}
& g\left(F_{P x, Q y}(t)\right) \\
& \quad \leq \phi\left(\max \left\{\begin{array}{c}
g\left(F_{A B x, S T y}(t)\right)+g\left(F_{P x, A B x}(t)\right)+g\left(F_{Q y, S T y}(t)\right), \\
g\left(F_{P x, A B x}(t)\right)+g\left(F_{Q y, A B x}(t)\right), \\
g\left(F_{P x, S T y}(t)\right)+g\left(F_{Q y, S T y}(t)\right)
\end{array}\right\}\right)
\end{aligned}
$$


for all $x, y \in X$ and $t>0$, where a function $\phi:[0, \infty) \rightarrow[0, \infty)$ satisfies the condition $(\Phi)$,

(d) $A B=B A, S T=T S, P B=B P, Q T=T Q$,

(e) either $P$ or $A B$ is continuous,

(f) the pairs $(P, A B)$ and $(Q, S T)$ are mutually compatible of type $(A)$.

Then $A, B, P, Q, S$ and $T$ have a unique common fixed point.

Proof. Let $x_{0}$ be an arbitrary point of $X$. By (a), there exists $x_{1}, x_{2} \in X$ such that $P x_{0}=S T x_{1}=y_{0}$ and $Q x_{1}=A B x_{1}=y_{1}$. Inductively, we can construct sequences $\left\{x_{n}\right\}$ and $\left\{y_{n}\right\}$ in $X$ such that $P x_{2 n}=S T x_{2 n+1}=y_{2 n}$ and $Q x_{2 n+1}=A B x_{2 n+2}=y_{2 n+1}$ for $n=0,1,2, \ldots$

Step1. We shall show that the sequence $\left\{y_{n}\right\}$ is a Cauchy sequence.

Since $P x_{2 n}=S T x_{2 n+1}$, using $(\mathrm{b})$, we have $g\left(F_{y_{2 n} y_{2 n+1}}(t)\right)=$ $g\left(F_{P x_{2 n} Q y_{2 n+1}}(t)\right) \leq \phi\left(g\left(F_{y_{2 n} y_{2 n+1}}(t)\right)\right)$ and since $Q x_{2 n+1}=A B x_{2 n+2}$, we also have $g\left(F_{y_{2 n}, y_{2 n-1}}(t)\right)=g\left(F_{P x_{2 n}, Q y_{2 n-1}}(t)\right) \leq \phi\left(g\left(F_{y_{2 n-1}, y_{2 n+2}}(t)\right)\right)$. Thus $g\left(F_{y_{n}, y_{n+1}}(t)\right) \leq \phi\left(g\left(F_{y_{n-1}, y_{n}}(t)\right)\right)$ for $n=1,2, \ldots$ Hence $g\left(F_{y_{n}, y_{n+1}}(t)\right) \leq$ $\phi^{n}\left(g\left(F_{y_{0}, y_{1}}(t)\right)\right)$ for $n=1,2, \ldots$ Therefore, from Lemma 1 ,

$$
g\left(F_{y_{n}, y_{n+1}}(t)\right) \rightarrow 0 \text { as } n \rightarrow \infty \text {. }
$$

Suppose $\left\{y_{n}\right\}$ is not a Cauchy sequence. Since $g$ is strictly decreasing, from Lemma 2 , there exist $\varepsilon_{0}>0, t_{0}>0$ and two sequences $\left\{m_{k}\right\},\left\{n_{k}\right\}$ of positive integers such that

(a) $m_{k}>n_{k}+1$ and $n_{k} \rightarrow \infty$ as $k \rightarrow \infty$,

(b) $g\left(F_{y_{m_{k}}, y_{n_{k}}}\left(t_{0}\right)\right)>g\left(1-\varepsilon_{0}\right)$ and $g\left(F_{y_{m_{k}-1}, y_{n_{k}}}\left(t_{0}\right)\right) \leq g\left(1-\varepsilon_{0}\right)$ for $k=1,2, \ldots$

Therefore

$$
\begin{aligned}
g\left(1-\varepsilon_{0}\right) & <g\left(F_{y_{m_{k}}, y_{n_{k}}}\left(t_{0}\right)\right) \\
& \leq g\left(F_{y_{m_{k}}, y_{m_{k}-1}}\left(t_{0}\right)\right)+g\left(F_{y_{m_{k}-1}, y_{n_{k}}}\left(t_{0}\right)\right) \\
& \leq g\left(F_{y_{m_{k}}, y_{m_{k}-1}}\left(t_{0}\right)\right)+g\left(1-\varepsilon_{0}\right)
\end{aligned}
$$

and letting $k \rightarrow \infty$, we have

$$
\lim _{n \rightarrow \infty} g\left(F_{y_{m_{k}}, y_{n_{k}}}\left(t_{0}\right)\right)=g\left(1-\varepsilon_{0}\right)
$$

On the other hand, we have

$$
\begin{aligned}
g\left(1-\varepsilon_{0}\right) & <g\left(F_{y_{m_{k}}, y_{n_{k}}}\left(t_{0}\right)\right) \\
& \leq g\left(F_{y_{m_{k}}, y_{n_{k}+1}}\left(t_{0}\right)\right)+g\left(F_{y_{n_{k}+1}, y_{n_{k}}}\left(t_{0}\right)\right) .
\end{aligned}
$$

Without loss of generality assume that both $m_{k}$ and $n_{k}$ are even. Using (c), 
we have

$$
\begin{aligned}
g\left(F_{y_{m_{k}}, y_{n_{k}+1}}\left(t_{0}\right)\right) & =g\left(F_{P x_{m_{k}}, Q x_{m_{k}+1}}\left(t_{0}\right)\right) \\
& \leq \phi\left(\max \left\{\begin{array}{c}
g\left(F_{y_{m_{k}-1}, y_{n_{k}}}\left(t_{0}\right)\right)+g\left(F_{y_{m_{k}}, y_{m_{k}-1}}\left(t_{0}\right)\right) \\
+g\left(F_{y_{n_{k}+1}, y_{n_{k}}}\left(t_{0}\right)\right), \\
g\left(F_{y_{m_{k}}, y_{m_{k}-1}}\left(t_{0}\right)\right)+g\left(F_{y_{m_{k}-1}, y_{n_{k}+1}}\left(t_{0}\right)\right), \\
g\left(F_{y_{n_{k}+1}, y_{n_{k}}}\left(t_{0}\right)\right)+g\left(F_{y_{m_{k}}, y_{n_{k}}}\left(t_{0}\right)\right)
\end{array}\right)\right) \\
& \leq \phi\left(\max \left\{\begin{array}{c}
g\left(1-\varepsilon_{0}\right)+g\left(F_{y_{m_{k}}, y_{m_{k}-1}}\left(t_{0}\right)\right) \\
+g\left(F_{y_{n_{k}+1}, y_{n_{k}}}\left(t_{0}\right)\right), \\
g\left(F_{y_{m_{k}}, y_{m_{k}-1}}\left(t_{0}\right)\right)+g\left(1-\varepsilon_{0}\right) \\
+g\left(F_{y_{n_{k}}, y_{n_{k}+1}}\left(t_{0}\right)\right), \\
g\left(F_{y_{n_{k}+1}, y_{n_{k}}}\left(t_{0}\right)\right)+g\left(F_{y_{m_{k}}, y_{n_{k}}}\left(t_{0}\right)\right)
\end{array}\right)\right)
\end{aligned}
$$

Substituting this in (3.3), letting $k \rightarrow \infty$ and using (3.1) and (3.2), we have

$$
g\left(1-\varepsilon_{0}\right) \leq \phi\left(g\left(1-\varepsilon_{0}\right)\right)<g\left(1-\varepsilon_{0}\right)
$$

which is a contradiction. Hence $\left\{y_{n}\right\}$ is a Cauchy sequence. Since $(X, F, *)$ is complete, it converges to a point $z$ in $X$. Also its subsequences converge as follows: $\left\{P x_{2 n}\right\} \rightarrow z,\left\{A B x_{2 n}\right\} \rightarrow z,\left\{Q x_{2 n+1}\right\} \rightarrow z$ and $\left\{S T x_{2 n+1}\right\} \rightarrow z$.

Case I. $A B$ is continuous, and $(P, A B)$ and $(Q, S T)$ are compatible of type $(A-1)$.

Since $A B$ is continuous, $A B(A B) x_{2 n} \rightarrow A B z$ and $(A B) P x_{2 n} \rightarrow A B z$. Since $(P, A B)$ is compatible of type (A-1), $P P x_{2 n} \rightarrow A B z$.

Step 2. By taking $x=P x_{2 n}, y=x_{2 n+1}$ in (c), we have

$$
\begin{array}{r}
g\left(F_{P P x_{2 n}, Q x_{2 n+1}}(t)\right) \\
\leq \phi\left(\max \left\{\begin{array}{c}
g\left(F_{(A B) P x_{2 n}, S T x_{2 n+1}}(t)\right)+g\left(F_{P P x_{2 n},(A B) P x_{2 n}}(t)\right) \\
+g\left(F_{Q x_{2 n+1}, S T x_{2 n+1}}(t)\right), \\
g\left(F_{P P x_{2 n},(A B) P x_{2 n}}(t)\right)+g\left(F_{Q x_{2 n+1},(A B) P x_{2 n}}(t)\right), \\
g\left(F_{P P x_{2 n}, S T x_{2 n+1}}(t)\right)+g\left(F_{Q x_{2 n+1}, S T x_{2 n+1}}(t)\right)
\end{array}\right\}\right)
\end{array}
$$

this implies that, as $n \rightarrow \infty$

$$
\begin{aligned}
g\left(F_{A B z, z}(t)\right) & \leq \phi\left(\max \left\{\begin{array}{c}
g\left(F_{A B z, z}(t)\right)+g\left(F_{A B z, A B z}(t)\right)+g\left(F_{z, z}(t)\right), \\
g\left(F_{A B z, A B z}(t)\right)+g\left(F_{z, A B z}(t)\right), \\
g\left(F_{z, z}(t)\right)+g\left(F_{z, z}(t)\right)
\end{array}\right)\right) \\
& =\phi\left(g\left(F_{A B z, z}(t)\right)\right)
\end{aligned}
$$

which means that, by Lemma $1, g\left(F_{A B z, z}(t)\right)=0$ for all $t>0$ and it follows that $z=A B z$. 
Step 3. By taking $x=z, y=x_{2 n+1}$ in (c), we have

$$
g\left(F_{P z, Q x_{2 n+1}}(t)\right)\left(\max \left\{\begin{array}{c}
g\left(F_{A B z, S T x_{2 n+1}}(t)\right)+g\left(F_{P z, A B z}(t)\right) \\
+g\left(F_{Q x_{2 n+1}, S T x_{2 n+1}}(t)\right), \\
g\left(F_{P z, A B z}(t)\right)+g\left(F_{Q x_{2 n+1}, A B z}(t)\right), \\
g\left(F_{P z, S T x_{2 n+1}}(t)\right)+g\left(F_{Q x_{2 n+1}, S T x_{2 n+1}}(t)\right)
\end{array}\right\}\right)
$$

this implies that, as $n \rightarrow \infty$

$$
\begin{aligned}
g\left(F_{P z, z}(t)\right) & \leq \phi\left(\max \left\{\begin{array}{c}
g\left(F_{z, z}(t)\right)+g\left(F_{P z, z}(t)\right)+g\left(F_{z, z}(t)\right) \\
g\left(F_{P z, z}(t)\right)+g\left(F_{z, z}(t)\right), \\
g\left(F_{P z, z}(t)\right)+g\left(F_{z, z}(t)\right)
\end{array}\right\}\right) \\
& =\phi\left(g\left(F_{P z, z}(t)\right)\right)
\end{aligned}
$$

which means that $z=P z$. Therefore, $z=A B z=P z$.

Step 4. By taking $x=B z, y=x_{2 n+1}$ in (c) and using (d), we have

$$
\begin{aligned}
& g\left(F_{P(B z), Q x_{2 n+1}}(t)\right) \\
& \leq \phi\left(\max \left\{\begin{array}{c}
g\left(F_{A B(B z), S T x_{2 n+1}}(t)\right)+g\left(F_{P(B z), A B(B z)}(t)\right) \\
+g\left(F_{Q x_{2 n+1}, S T x_{2 n+1}}(t)\right), \\
g\left(F_{P(B z), A B(B z)}(t)\right)+g\left(F_{Q x_{2 n+1}, A B(B z)}(t)\right), \\
g\left(F_{P(B z), S T x_{2 n+1}}(t)\right)+g\left(F_{Q x_{2 n+1}, S T x_{2 n+1}}(t)\right)
\end{array}\right\}\right)
\end{aligned}
$$

this implies that, as $n \rightarrow \infty$

$$
\begin{aligned}
g\left(F_{B z, z}(t)\right) & \leq \phi\left(\max \left\{\begin{array}{c}
g\left(F_{B z, z}(t)\right)+g\left(F_{B z, B z}(t)\right)+g\left(F_{z, z}(t)\right) \\
g\left(F_{B z, B z}(t)\right)+g\left(F_{z, B z}(t)\right), \\
g\left(F_{B z, z}(t)\right)+g\left(F_{z, z}(t)\right)
\end{array}\right\}\right) \\
& =\phi\left(g\left(F_{B z, z}(t)\right)\right)
\end{aligned}
$$

which means that $z=B z$. Since $z=A B z$, we have $z=A z$. Therefore, $z=A z=B z=P z$.

Step 5. Since $P(X) \subseteq S T(X)$, there exists $w \in X$ such that $z=P z=$ $S T w$. By taking $x=x_{2 n}, y=w$ in (c), we have

$$
g\left(F_{P x_{2 n}, Q w}(t)\right) \leq \phi\left(\max \left\{\begin{array}{c}
g\left(F_{A B x_{2 n}, S T w}(t)\right)+g\left(F_{P x_{2 n}, A B x_{2 n}}(t)\right) \\
+g\left(F_{Q w, S T w}(t)\right) \\
g\left(F_{P x_{2 n}, A B x_{2 n}}(t)\right)+g\left(F_{Q w, A B x_{2 n}}(t)\right), \\
g\left(F_{P x_{2 n}, S T w}(t)\right)+g\left(F_{Q w, S T w}(t)\right)
\end{array}\right\}\right)
$$


this implies that, as $n \rightarrow \infty$

$$
\begin{aligned}
g\left(F_{z, Q w}(t)\right) & \leq \phi\left(\max \left\{\begin{array}{c}
g\left(F_{z, z}(t)\right)+g\left(F_{z, z}(t)\right)+g\left(F_{Q w, z}(t)\right), \\
g\left(F_{z, z}(t)\right)+g\left(F_{Q w, z}(t)\right), \\
g\left(F_{z, z}(t)\right)+g\left(F_{Q w, z}(t)\right)
\end{array}\right\}\right) \\
& =\phi\left(g\left(F_{z, Q w}(t)\right)\right)
\end{aligned}
$$

which means that $z=Q w$. Hence, $S T w=z=Q w$. Since $(Q, S T)$ is compatible of type (A-1), we have $Q(S T) w=S T(S T) w$. Thus, $S T z=Q z$.

Step 6. By taking $x=x_{2 n}, y=z$ in (c) and using Step 5, we have

$$
g\left(F_{P_{x_{2 n}, Q z}}(t)\right) \leq \phi\left(\max \left\{\begin{array}{c}
g\left(F_{A B x_{2 n}, S T z}(t)\right)+g\left(F_{P x_{2 n}, A B x_{2 n}}(t)\right) \\
+g\left(F_{Q z, S T z}(t)\right), \\
g\left(F_{P x_{2 n}, A B x_{2 n}}(t)\right)+g\left(F_{Q z, A B x_{2 n}}(t)\right), \\
g\left(F_{P x_{2 n}, S T z}(t)\right)+g\left(F_{Q z, S T z}(t)\right)
\end{array}\right\}\right)
$$

this implies that, as $n \rightarrow \infty$

$$
\begin{aligned}
g\left(F_{z, Q z}(t)\right) & \leq \phi\left(\max \left\{\begin{array}{c}
g\left(F_{z, Q z}(t)\right)+g\left(F_{z, z}(t)\right)+g\left(F_{Q z, Q z}(t)\right), \\
g\left(F_{z, z}(t)\right)+g\left(F_{Q z, z}(t)\right), \\
g\left(F_{z, Q z}(t)\right)+g\left(F_{Q z, Q z}(t)\right)
\end{array}\right\}\right) \\
& =\phi\left(g\left(F_{z, Q z}(t)\right)\right)
\end{aligned}
$$

which means that $z=Q z$. Since $S T z=Q z$, we have $z=S T z$. Therefore, $z=A z=B z=P z=Q z=S T z$.

Step 7. By taking $x=x_{2 n}, y=T z$ in (c) and using (d), we have

$$
g\left(F_{P x_{2 n}, T z}(t)\right) \leq \phi\left(\max \left\{\begin{array}{c}
g\left(F_{A B x_{2 n}, S T(T z)}(t)\right)+g\left(F_{P x_{2 n}, A B x_{2 n}}(t)\right) \\
+g\left(F_{Q(T z), S T(T z)}(t)\right), \\
g\left(F_{P x_{2 n}, A B x_{2 n}}(t)\right)+g\left(F_{Q(T z), A B x_{2 n}}(t)\right), \\
g\left(F_{P x_{2 n}, S T(T z)}(t)\right)+g\left(F_{Q(T z), S T(T z)}(t)\right)
\end{array}\right\}\right)
$$

this implies that, as $n \rightarrow \infty$

$$
\begin{aligned}
g\left(F_{z, T z}(t)\right) & \leq \phi\left(\max \left\{\begin{array}{c}
g\left(F_{z, T z}(t)\right)+g\left(F_{z, z}(t)\right)+g\left(F_{T z, T z}(t)\right), \\
g\left(F_{z, z}(t)\right)+g\left(F_{T z, z}(t)\right), \\
g\left(F_{z, T z}(t)\right)+g\left(F_{T z, T z}(t)\right)
\end{array}\right\}\right) \\
& =\phi\left(g\left(F_{z, T z}(t)\right)\right)
\end{aligned}
$$

which means that $z=T z$. Since $z=S T z$, we have $z=S z$. Therefore, $z=A z=B z=P z=Q z=S z=T z$, that is, $z$ is the common fixed point of $A, B, P, Q, S$ and $T$. 
Similarly, it is clear that $z$ is also the common fixed point of $A, B, P, Q, S$ and $T$ in the case $A B$ is continuous, and $(P, A B)$ and $(Q, S T)$ are compatible of type (A-2).

Case II. $P$ is continuous, and $(P, A B)$ and $(Q, S T)$ are compatible of type (A-1).

Since $P$ is continuous, $P P x_{2 n} \rightarrow P z$ and $P(A B) x_{2 n} \rightarrow P z$. Since $(P, A B)$ is compatible of type (A-1), $A B(A B) x_{2 n} \rightarrow P z$.

Step 8. By taking $x=A B x_{2 n}, y=x_{2 n+1}$ in (c), we have

$$
\begin{aligned}
& g\left(F_{P(A B) x_{2 n}, Q x_{2 n+1}}(t)\right) \\
\leq & \left.\left(\max \left\{\begin{array}{c}
g\left(F_{A B(A B) x_{2 n}, S T x_{2 n+1}}(t)\right)+g\left(F_{P(A B) x_{2 n}, A B(A B) x_{2 n}}(t)\right) \\
+g\left(F_{Q x_{2 n+1}, S T x_{2 n+1}}(t)\right), \\
g\left(F_{P(A B) x_{2 n}, A B(A B) x_{2 n}}(t)\right)+g\left(F_{Q x_{2 n+1}, A B(A B) x_{2 n}}(t)\right), \\
g\left(F_{P(A B) x_{2 n}, S T x_{2 n+1}}(t)\right)+g\left(F_{Q x_{2 n+1}, S T x_{2 n+1}}(t)\right)
\end{array}\right\}\right)\right)
\end{aligned}
$$

this implies that, as $n \rightarrow \infty$

$$
\begin{aligned}
g\left(F_{P z, z}(t)\right) & \leq \phi\left(\max \left\{\begin{array}{c}
g\left(F_{P z, z}(t)\right)+g\left(F_{P z, P z}(t)\right)+g\left(F_{z, z}(t)\right) \\
g\left(F_{P z, P z}(t)\right)+g\left(F_{z, P z}(t)\right), \\
g\left(F_{P z, z}(t)\right)+g\left(F_{z, z}(t)\right)
\end{array}\right\}\right) \\
& =\phi\left(g\left(F_{P z, z}(t)\right)\right)
\end{aligned}
$$

which means that $z=P z$. Now using Step 5-7, we have $z=Q z=S T z=$ $S z=T z$.

Step 9. Since $Q(X) \subseteq A B(X)$, there exists $w \in X$ such that $z=Q z=$ $A B w$. By taking $x=w, y=x_{2 n+1}$ in (c), we have

$$
g\left(F_{P w, Q x_{2 n+1}}(t)\right)\left(\max \left\{\begin{array}{c}
g\left(F_{A B w, S T x_{2 n+1}}(t)\right)+g\left(F_{P w, A B w}(t)\right) \\
+g\left(F_{Q x_{2 n+1}, S T x_{2 n+1}}(t)\right), \\
g\left(F_{P w, A B w}(t)\right)+g\left(F_{Q x_{2 n+1}, A B w}(t)\right), \\
g\left(F_{P w, S T x_{2 n+1}}(t)\right)+g\left(F_{Q x_{2 n+1}, S T x_{2 n+1}}(t)\right)
\end{array}\right)\right)
$$

this implies that, as $n \rightarrow \infty$

$$
\begin{aligned}
g\left(F_{P w, z}(t)\right) & \leq \phi\left(\max \left\{\begin{array}{c}
g\left(F_{z, z}(t)\right)+g\left(F_{P w, z}(t)\right)+g\left(F_{z, z}(t)\right), \\
g\left(F_{P w, z}(t)\right)+g\left(F_{z, z}(t)\right), \\
g\left(F_{P w, z}(t)\right)+g\left(F_{z, z}(t)\right)
\end{array}\right\}\right) \\
& =\phi\left(g\left(F_{P w, z}(t)\right)\right)
\end{aligned}
$$

which means that $z=P w$. Since $z=Q z=A B w, P w=A B w$. Since $(P, A B)$ is compatible of type (A-1), we have $P z=A B z$. Also $z=B z$ 
follows from Step 4. Thus, $z=A z=B z=P z$. Hence, $z$ is the common fixed point of the six maps in this case also.

Similarly, it is clear that $z$ is also the common fixed point of $A, B, P, Q, S$ and $T$ in the case $P$ is continuous, and $(P, A B)$ and $(Q, S T)$ are compatible of type (A-2).

Step 10. For uniqueness, let $v(v \neq z)$ be another common fixed point of $A, B, P, Q, S$ and $T$. Taking $x=z, y=v$ in (c), we have

$$
\begin{aligned}
& g\left(F_{P z, Q v}(t)\right) \\
& \leq \phi\left(\max \left\{\begin{array}{c}
g\left(F_{A B z, S T v}(t)\right)+g\left(F_{P z, A B z}(t)\right)+g\left(F_{Q v, S T v}(t)\right), \\
g\left(F_{P z, A B z}(t)\right)+g\left(F_{Q v, A B z}(t)\right), \\
g\left(F_{P z, S T v}(t)\right)+g\left(F_{Q v, S T v}(t)\right)
\end{array}\right\}\right)
\end{aligned}
$$

which implies that

$$
\begin{aligned}
g\left(F_{z, v}(t)\right) & \leq \phi\left(\max \left\{\begin{array}{c}
g\left(F_{z, v}(t)\right)+g\left(F_{z, z}(t)\right)+g\left(F_{v, v}(t)\right), \\
g\left(F_{z, z}(t)\right)+g\left(F_{v, z}(t)\right), \\
g\left(F_{z, v}(t)\right)+g\left(F_{v, v}(t)\right)
\end{array}\right\}\right) \\
& =\phi\left(g\left(F_{z, v}(t)\right)\right)
\end{aligned}
$$

so we have $z=v$. This completes the proof of the theorem.

If we take $A=B=S=T=I_{X}$ (the identity map on $X$ ) in Theorem 1, we have the following:

Corollary 1. Let $P$ and $Q$ be self maps on a complete $N$. A. Menger $P M$-space $(X, F, *)$. If $g\left(F_{P x, Q y}(t)\right) \leq \phi\left(g\left(F_{x, y}(t)\right)\right)$ and

$$
g\left(F_{P x, Q y}(t)\right) \leq \phi\left(\max \left\{\begin{array}{c}
g\left(F_{x, y}(t)\right)+g\left(F_{P x, x}(t)\right)+g\left(F_{Q y, y}(t)\right), \\
g\left(F_{P x, x}(t)\right)+g\left(F_{Q y, x}(t)\right), \\
g\left(F_{P x, y}(t)\right)+g\left(F_{Q y, y}(t)\right)
\end{array}\right\}\right)
$$

for all $x, y \in X$ and $t>0$, where a function $\phi:[0, \infty) \rightarrow[0, \infty)$ satisfies the condition $(\Phi)$, then $P$ and $Q$ have a unique common fixed point.

In [7], Sehgal and Bharucha-Reid presented the probabilistic version of the Banach contraction theorem. Next we prove such theorem for N. A. Menger PM-spaces as follows:

Corollary 2. Let $P$ be self maps on a complete N.A. Menger PM-space $(X, F, *)$. If

$$
g\left(F_{P x P y}(t)\right) \leq \phi\left(g\left(F_{x y}(t)\right)\right)
$$

for all $x, y \in X$ and $t>0$, then $P$ has a unique common fixed point. 
Proof. The proof follows from Corollary 1 since $P=Q$ and

$$
g\left(F_{x y}(t)\right)=\max \left\{g\left(F_{x y}(t)\right), g\left(F_{x P x}(t)\right), g\left(F_{y Q y}(t)\right), g\left(F_{y P x}(t)\right), g\left(F_{x Q y}(t)\right)\right\} .
$$

EXAMPLE 2. Let $(X, d)$ be a metric space with the usual metric $d$ where $X=[0,1]$ and $(X, F, *)$ be the induced N. A. Menger PM-space with $g(t)=$ $1-t$ and $F_{x y}(t)=H(t-d(x, y))$ for all $x, y \in X, t>0$. Let $A, B, P, Q, S$ and $T$ be maps from $X$ into itself defined as

$$
A x=x / 5, B x=x / 3, P x=x / 6, Q x=0, S x=x, T x=x / 2
$$

for all $x \in X$. Then

$$
P(X)=\left[0, \frac{1}{6}\right] \subset\left[0, \frac{1}{2}\right]=S T(X)
$$

and

$$
Q(X)=\{0\} \subset\left[0, \frac{1}{15}\right]=A B(X) .
$$

If we take $t=1$ and $\alpha=1$, we see that the condition (b) and (c) of the main Theorem is satisfied. Clearly, conditions (d) and (e) of the main Theorem are also satisfied. Moreover, the pairs $(P, A B)$ and $(Q, S T)$ are mutually compatible of type (A). In fact, if $\lim _{n \rightarrow \infty} x_{n}=0$, where $\left\{x_{n}\right\}$ is a sequence in $X$ such that $\lim _{n \rightarrow \infty} P x_{n}=\lim _{n \rightarrow \infty} A B x_{n}=0$ and $\lim _{n \rightarrow \infty} Q x_{n}=$ $\lim _{n \rightarrow \infty} S T x_{n}=0$ for some $0 \in X$, then

$$
\lim _{n \rightarrow \infty} g\left(F_{P(A B) x_{n}, A B(A B) x_{n}}(t)\right)=g(H(t))=0
$$

and

$$
\lim _{n \rightarrow \infty} g\left(F_{(A B) P x_{n}, P P x_{n}}(t)\right)=g(H(t))=0
$$

hence $(P, A B)$ and $(Q, S T)$ are compatible of type (A-1). Similarly, the pairs $(A B, P)$ and $(S T, Q)$ are also compatible of type (A-2). Thus, all conditions of the main Theorem are satisfied and 0 is the unique common fixed point of $A, B, P, Q, S$ and $T$.

\section{References}

[1] S. S. Chang, Fixed point theorems for single-valued and multi-valued mappings in nonArchimedean Menger probabilistic metric spaces, Math. Japon. 35 (5) (1990), 875-885.

[2] Y. J. Cho, K. S. Ha, S. S. Chang, Common fixed point theorems for compatible maps of type (A) in non-Archimedean Menger PM-spaces, Math. Japon. 46 (1) (1997), 169-179.

[3] Y. J. Cho, H. K. Pathak, S. M. Kang, J. S. Jung, Common fixed points of compatible maps of type $(\beta)$ on fuzzy metric spaces, Fuzzy Sets and Systems 93 (1998), 99-111.

[4] O. Hadzic, $A$ note on Istratescu's fixed point theorem in non-Archimedean Menger spaces, Bull. Math. Soc. Sci. Math. R. S. Roumanie 24 (72) (1980), 277-280. 
[5] S. Kutukcu, C. Yildiz, A common fixed point theorem of compatible and weak compatible maps on Menger spaces, Kochi Math. J. (2008), to appear in vol. 3.

[6] B. Schweizer, A. Sklar, Statistical metric spaces, Pacific J. Math. 10 (1960), 313-324.

[7] V. M. Sehgal, A. T. Bharucha-Reid, Fixed point of contraction mapping on PM spaces, Math. Systems Theory 6 (1972), 97-100.

\author{
Servet Kutukcu \\ DEPARTMENT OF MATHEMATICS \\ FACULTY OF SCIENCE AND ARTS \\ ONDOKUZ MAYIS UNIVERSITY \\ 55139 KURUPELIT, SAMSUN, TURKEY \\ E-mail: skutukcu@yahoo.com \\ Sushil Sharma \\ DEPARTMENT OF MATHEMATICS \\ MADHAV SCIENCE COLLEGE \\ UJJAIN, M.P. 456 001, INDIA \\ E-mail: sksharma2005@yahoo.com
}

Received October 19, 2008. 
